\title{
Long-term Outcomes of Direct Endoscopic Necrosectomy for Complicated or Symptomatic Walled-Off Necrosis: A Korean Multicenter Study
}

\author{
Yeon Suk Kim¹, Jae Hee $\mathrm{Cho}^{2}$, Dong Hui Cho ${ }^{3}$, Se Woo Park ${ }^{4}$ Sung-Hoon Moon ${ }^{5}$, Jin-Seok Park ${ }^{6}$, Yun Nah Lee ${ }^{7}$, \\ and Sang Soo Lee $^{3}$ \\ 1Division of Gastroenterology, Department of Internal Medicine, Gil Medical Center, Gachon University College of Medicine, Incheon, \\ ${ }^{2}$ Division of Gastroenterology, Department of Internal Medicine, Gangnam Severance Hospital, Yonsei University College of Medicine, \\ ${ }^{3}$ Department of Gastroenterology, Asan Medical Center, University of Ulsan College of Medicine, Seoul, ${ }^{4}$ Division of Gastroenterology, \\ Department of Internal Medicine, Hallym University Dongtan Sacred Heart Hospital, Hallym University College of Medicine, \\ Hwaseong, ${ }^{5}$ Department of Internal Medicine, Hallym University Sacred Heart Hospital, Hallym University College of Medicine, \\ Anyang, ${ }^{6}$ Digestive Disease Center, Department of Internal Medicine, Inha University School of Medicine, Incheon, and ${ }^{7}$ Digestive \\ Disease Center, Department of Internal Medicine, Soonchunhyang University School of Medicine, Bucheon, Korea
}

\section{Article Info}

Received September 30, 2020

Revised December 28, 2020

Accepted January 8, 2021

\section{Corresponding Author}

Jae Hee Cho

ORCID https://orcid.org/0000-0003-4174-0091

E-mail jhcho9328@yuhs.ac

\section{Sang Soo Lee}

ORCID https://orcid.org/0000-0002-3785-2341

E-mail ssleedr@amc.seoul.kr

\begin{abstract}
Background/Aims: The endoscopic step-up approach is accepted as the preferred treatment for complicated or symptomatic walled-off necrosis (WON). Direct endoscopic necrosectomy (DEN) is an effective therapeutic option, but few reports describe long-term follow-up in this patient population. Thus, we aim to assess the long-term outcomes of DEN following severe necrotizing pancreatitis.
\end{abstract}

Methods: The data of all acute pancreatitis patients who underwent DEN following endoscopic transmural drainage from six referral centers between 2007 and 2017 were retrospectively collected.

Results: Sixty patients ( $76.7 \%$ male, mean age 48.3 years) underwent a median of 4 sessions of DEN starting at a median of 45.5 days after the onset of acute pancreatitis. Clinical success was achieved in 51 patients (85\%), with a $35 \%$ complication rate and a $5 \%$ mortality rate. Using multivariate analysis, the risk factor associated with DEN failure or major DEN complications requiring intervention or surgery was an identified bacterial/fungal WON infection (odds ratio, 19.3; 95\% confidence interval, 1.5 to 261.7). During the median follow-up period of 27 months, complicated WON recurrence was observed in $5.3 \%$ of patients, and long-term complications occurred in $24.6 \%$ of patients (four exocrine insufficiency, nine newly developed diabetes mellitus, one recurrent small bowel obstruction, one chylous ascites).

Conclusions: Considering that long-term complications are similar to those observed after pancreatectomy, DEN should be performed meticulously while minimizing damage to the viable pancreatic parenchyma with adequate antibiotic escalation. (Gut Liver 2021;15:930-939)

Key Words: Acute pancreatitis; Infected necrosis; Necrosectomy; Endoscopy; Antibiotics

\section{INTRODUCTION}

Acute necrotizing pancreatitis is a potentially lethal disease, and its incidence is currently increasing. Because infected necrotizing pancreatitis is associated with a mortality of $20 \%$ to $30 \%$, an expert multidisciplinary team approach is required to improve clinical outcomes. ${ }^{1-5}$ Over the past decade, much progress has been made in improving the management of acute necrotizing pancreatitis. ${ }^{6,7}$ A surgical step-up approach consisting of percutaneous catheter drainage followed by minimally invasive surgical necrosectomy has replaced open necrosectomy as the standard treatment. ${ }^{8-10}$ Endoscopic treatment has also been highlighted because it is potentially less invasive and can 
also be performed in a step-up manner. ${ }^{11-13}$ Endoscopic transmural drainage (ETD) is usually the first procedure to be performed, followed up by direct endoscopic necrosectomy (DEN) if required for improving clinical outcomes. In a Dutch randomized trial, the risk of developing a pancreatic fistula and the length of hospital stay were lower in the endoscopy group when compared to the surgical group. However, endoscopy did not demonstrate superior therapeutic outcomes in terms of mortality and major complications. ${ }^{14}$ Contrary to these data, a U.S. randomized trial indicated that an endoscopic transluminal approach reduced major complications, lowered costs, and increased the patients' quality-of-life when compared to minimally invasive surgery. ${ }^{15,16}$ Controversy remains as to whether an endoscopic step-up approach should be used as the preferred therapeutic modality for infected necrotizing pancreatitis. Furthermore, sufficient information from long-term follow-up after DEN does not currently exist. Therefore, we aimed to assess the long-term outcomes of DEN following ETD as part of an endoscopic step-up approach in patients with severe acute necrotizing pancreatitis.

\section{MATERIALS AND METHODS}

\section{Patient information}

The Korean Pancreatobiliary Association conducted a nationwide multicenter retrospective study by accumulating information on severe acute necrotizing pancreatitis patients who underwent DEN across six referral centers in Korea between January 2007 and December 2017. After standardized data collection forms were sent to each center, each institution retrospectively reviewed their database and collected the clinical data. The participating institutions were Asan Medical Center (Seoul), Gachon University Gil Medical Center (Incheon), Hallym University Dongtan Sacred Heart Hospital (Hwaseong), Hallym University Sacred Heart Hospital (Anyang), Inha University hospital (Incheon), and Soonchunhyang University Bucheon Hospital (Bucheon) in the Republic of Korea. Institutional review board approval was obtained for each participant in this study. The informed consent was waived. A total of 60 patients were ultimately included in this study.

\section{Procedures}

The indications for endoscopic intervention were determined for each patient. Inclusion criteria were symptomatic or complicated walled-off necrosis (WON), such as suspected/identified infection, and biliary/gastric outlet obstruction following severe necrotizing pancreatitis. The standard technique used for the endoscopic step-up ap- proach is described as follows. Endoscopic ultrasoundguided transmural drainage was performed using either one or two 7-F double-pigtail plastic stents (DPPS) or self-expandable metallic stent (SEMS). Based on clinical decisions, an additional nasocystic drainage catheter was placed into the WON cavity if necessary. Types of SEMS used were as follows: tubular fully covered SEMS including 26 Soo stents (Standard Sci Tech Inc., Seoul, Korea), seven AL stents (Standard Sci Tech Inc.) and two Nagi stents (Taewoong Medical, Goyang, Korea), or lumen-apposing metallic stent of six SPAXUS stents (Taewoong Medical). If there was no clinical improvement following ETD, a DEN was performed. In most cases, an established fistula tract was dilated using a large balloon (maximal diameter, 12 to $20 \mathrm{~mm}$ ) several days after placement of stent or drainage catheter. A conventional forward-viewing endoscope or water jet scope (GIF-Q260J; Olympus, Tokyo, Japan) was subsequently advanced into the WON cavity and mechanical debridement of the necrotic tissue was performed using snares, forceps, and stone removal baskets accompanied by forceful irrigation using normal saline. In anticipation of a subsequent DEN, the fistula to the necrotic cavity is maintained by placing a DPPS or nasocystic drainage catheter. Repeated DEN sessions were carried out until clinical improvement was achieved, regardless of whether the necrotic debris in the WON was completely removed. At the final DEN session, a DPPS might be placed depending on each institution's protocol. Depending on the patient's condition, additional intervention measures, including transpapillary pancreatic duct (PD) stenting, percutaneous drainage, and surgery were performed as necessary. Additionally, in order to diagnose WON infection, microbial culture was conducted with pus aspirated in the ETD procedure. If the patient's condition did not improve, additional culture tests were performed in pus and necrotic tissue during DEN.

\section{Outcomes}

The primary outcome was to assess the long-term clinical outcomes, such as recurrence of the symptomatic/ complicated WON and late complications following DEN. The definition of symptomatic/complicated WON is as follows: (1) clinical suspicion or documented infected WON with clinical deterioration; (2) symptomatic sterile WON including intractable pain, persistent unwellness, ongoing gastrointestinal obstruction despite medical treatment; (3) abdominal compartment syndrome or disconnected PD syndrome with WON. In terms of long-term results, the recurrence of WON was defined as the case of WON redevelopment after 6 months of last DEN session in a patient who had completed treatment. New onset diabetes mellitus was defined as requiring insulin or oral antidia- 
betic drugs for at least 6 months after DEN and not present prior to the onset of pancreatitis. Exocrine insufficiency was defined as the development of clinical symptoms of steatorrhea and weight loss in patients not previously taking oral pancreatic enzyme supplements. The secondary outcomes were to evaluate the initial treatment success and early complication rates associated with DEN. Clinical success of the DEN was defined as improvement of WON on computed tomography (CT) scan in association with clinical resolution of symptoms at 6-month follow-up period. ${ }^{17}$ DEN failure was defined as the need for rescue surgery or death during index admission as well as readmission for management of recurred pancreatitis or WON within 6 months.

Other DEN-related complications are defined as follows; "massive bleeding" is the bleeding within the intraabdominal cavity or gastrointestinal tract requiring radiologic interventional procedure, "visceral perforation" is the perforation of visceral organ with intraabdominal free air, "duodenal or colonic fistula" is the formation of a fistula between the small bowel or colon and pancreas confirmed on imaging or endoscopy.

\section{Statistical analysis}

Differences of continuous variables were compared using the independent t-tests. Categorical variables were compared by the chi-square tests or Fisher exact test. Multivariate logistic regression analysis was performed to evaluate the independent effect of each variable. Odds ratios and confidence intervals were calculated. Statistical analysis was performed using the SPSS software, version 24.0 for Windows (IBM Corp., Armonk, NY, USA). Statistical significance was reached when $\mathrm{p}$-values were below 0.05 .

\section{RESULTS}

\section{Characteristics of patients}

A total of 60 patients ( $76.7 \%$ male, mean age 48.3 years) with complicated or symptomatic WON were included from six centers. Twenty of the total 60 patients were treated in the intensive care unit. Continuous renal replacement therapy was administered in 10 patients, and one patient improved after receiving extracorporeal membrane oxygenation. The main causes underlying acute pancreatitis in these patients were alcohol (45\%) and the presence of gallstones (25\%). The size of the WON (mean of long axis $\times$ short axis) was estimated to be $145.9 \times 72.1 \mathrm{~mm}$ and the median severity index determined by CT was 6 . Coronal CT imaging was used to evaluate the lower margin of the WON, which was found to be an extension to the pelvic cavity in 15 patients and below the kidney in 34 patients. Twenty-one patients had undergone percutaneous catheter drainage. Additionally, the medians of laboratory findings in these patients at the time of DEN were as fol-

Table 1. Characteristics of Patients with Complicated or Symptomatic Walled-Off Necrosis Who Underwent Direct Endoscopic Necrosectomy

\begin{tabular}{|c|c|}
\hline Patients & Value $(n=60)$ \\
\hline Age, yr & $48.3 \pm 14.4$ \\
\hline \multicolumn{2}{|l|}{ Sex } \\
\hline Male & $46(76.7)$ \\
\hline Female & 14 (23.3) \\
\hline $\mathrm{BMI}, \mathrm{kg} / \mathrm{m}^{2}$ & $24.4 \pm 3.71$ \\
\hline Smoking & 23 (38.3) \\
\hline Alcohol & $41(68.3)$ \\
\hline \multicolumn{2}{|l|}{ Etiology } \\
\hline Alcohol & $27(45.0)$ \\
\hline Gallstone & $15(25.0)$ \\
\hline Post-ERCP & $6(10.0)$ \\
\hline Others* & $12(20.0)$ \\
\hline \multicolumn{2}{|l|}{ Diabetes mellitus } \\
\hline Yes & $12(20.0)$ \\
\hline No & $48(80.0)$ \\
\hline \multicolumn{2}{|c|}{ Initial management of pancreatitis } \\
\hline ICU care & 20 (33.3) \\
\hline CRRT/ECMO & $10(16.7) / 1(1.7)$ \\
\hline CT severity index score & $6(5-7)$ \\
\hline Previous placement of PCD & $21(35.0)$ \\
\hline \multicolumn{2}{|l|}{ Main locations of WON } \\
\hline Head & $7(11.7)$ \\
\hline Body and tail & 32 (53.3) \\
\hline Entire pancreas & $21(35.0)$ \\
\hline \multicolumn{2}{|l|}{ Lower margin of WON cavity } \\
\hline Above kidney/below kidney & $11(18.3) / 34$ (56.7) \\
\hline Extension to pelvic cavity & $15(25.0)$ \\
\hline \multicolumn{2}{|l|}{ No. of WON cavities } \\
\hline Single & $28(46.7)$ \\
\hline Multiple & 32 (53.3) \\
\hline \multicolumn{2}{|l|}{ Size, mm } \\
\hline Long axis & $145.9 \pm 50.6$ \\
\hline Short axis & $72.1 \pm 33.1$ \\
\hline \multicolumn{2}{|c|}{ Laboratory findings at the time of DEN } \\
\hline White blood cell, $/ \mathrm{mm}^{3}$ & $16,380(13,047-20,005)$ \\
\hline $\mathrm{CRP}, \mathrm{mg} / \mathrm{dL}$ & $10.6(0.4-30.7)$ \\
\hline Amylase, $\mathrm{U} / \mathrm{L}$ & $438(146-2,667)$ \\
\hline Lipase, U/L & $938(106-10,886)$ \\
\hline Creatinine, $\mathrm{mg} / \mathrm{dL}$ & $1.03(0.71-1.57)$ \\
\hline
\end{tabular}

Data are presented as mean $\pm S D$, number $(\%)$, or median (interquartile range).

$\mathrm{BMI}$, body mass index; ERCP, endoscopic retrograde cholangiopancreatography; ICU, intensive care unit; CRRT, continuous renal replacement therapy; ECMO, extracorporeal membrane oxygenation; $C T$, computed tomography; PCD, percutaneous catheter drainage; WON, walled-off necrosis; DEN, direct endoscopic necrosectomy; CRP, C-reactive protein.

*Others include hypertriglyceridemia-induced, malignancy, postoperative, and trauma. 
lows: white blood cell counts $16,380 / \mathrm{mm}^{3}$, amylase $438 \mathrm{U} /$ $\mathrm{L}$, lipase $938 \mathrm{U} / \mathrm{L}$, creatinine $1.03 \mathrm{mg} / \mathrm{dL}$, and C-reactive protein $10.6 \mathrm{mg} / \mathrm{dL}$ (Table 1).

\section{Initial outcomes of DEN}

Initial ETD using DPPS or SEMS were performed after a median duration of 34 days (interquartile range [IQR],
21 to 52.5 days) from the onset of pancreatitis. Of them, transmural SEMS placement was achieved in 41 patients (68.3\%). If the patient's condition did not improve even during ETD, DEN was administered after a median duration of 45.5 days (IQR, 31.25 to 70.75 days) after the index admission. Indication of DEN were as follows; suspected infection (fever and pain) in 40 patients, intractable pain in

Table 2. Procedural Details and Outcomes of Direct Endoscopic Necrosectomy

\begin{tabular}{|c|c|}
\hline Direct endoscopic necrosectomy & Value \\
\hline Time from onset of pancreatitis to initial EUS drainage, day & $34(21.0-52.5)$ \\
\hline \multicolumn{2}{|l|}{ Placed drainage method } \\
\hline DPPS and/or nasocystic drainage catheter & 19 (31.7) \\
\hline SEMS and/or nasocystic drainage catheter & 41 (68.3) \\
\hline Time from onset of pancreatitis to the first DEN, day & $45.5(31.25-70.75)$ \\
\hline \multicolumn{2}{|l|}{ Indications of DEN } \\
\hline Suspected infection & $40(66.7)$ \\
\hline Intractable pain & $18(30.0)$ \\
\hline Gastric outlet obstruction & 2 (3.3) \\
\hline \multicolumn{2}{|l|}{ Diameter of dilation for entry site of DEN, mm } \\
\hline $12-15$ & 35 (58.3) \\
\hline $16-20$ & $25(41.7)$ \\
\hline \multicolumn{2}{|l|}{ Route } \\
\hline Transgastric & 53 (88.3) \\
\hline Transduodenal & 5 (8.3) \\
\hline Both & 2 (3.3) \\
\hline \multicolumn{2}{|l|}{ Gateway } \\
\hline Single & $49(81.7)$ \\
\hline Multiple & $11(18.3)$ \\
\hline No. of DEN & $4(2-6)$ \\
\hline Procedural time of DEN in each session, min & $48(37.25-64.23)$ \\
\hline \multicolumn{2}{|l|}{ Insufflation during the procedure } \\
\hline Room air & $6(10.0)$ \\
\hline $\mathrm{CO}_{2}$ gas & $54(90.0)$ \\
\hline ERCP & $27(45.0)$ \\
\hline Identified main PD disruption & $15(25.0)$ \\
\hline Transpapillary PD stent & $17(28.3)$ \\
\hline Preprocedural intravenous antibiotics & $60(100)$ \\
\hline \multicolumn{2}{|l|}{ Identified infection in WON cavity } \\
\hline Yes & 44 (73.3) \\
\hline No & $16(26.7)$ \\
\hline \multicolumn{2}{|l|}{ Clinical outcomes of DEN as part of endoscopic step-up approach } \\
\hline Success & $51(85.0)$ \\
\hline \multicolumn{2}{|l|}{ Failure } \\
\hline Surgery/pancreatitis-related mortality* & $6(10.0) / 3(5.0)$ \\
\hline WON recurrence within 6 months & $4(6.7)$ \\
\hline \multicolumn{2}{|l|}{ Degree of cavity clearance at the end of DEN } \\
\hline Complete clearance & 8 (13.3) \\
\hline Incomplete clearance & $52(86.7)$ \\
\hline Procedure related complications during the treatment period of DEN & $21(35.0)$ \\
\hline Massive bleeding requiring radiologic intervention & $9(15.0)$ \\
\hline Visceral perforation & $3(5.0)$ \\
\hline Stent migration & $8(13.3)$ \\
\hline Duodenal or colonic fistula & $5(8.3)$ \\
\hline Others ${ }^{\dagger}$ & 2 (3.3) \\
\hline
\end{tabular}

Data are presented as median (interquartile range) or number (\%).

EUS, endoscopic ultrasound; DPPS, double-pigtail plastic stents; SEMS, self-expandable metallic stent; DEN, direct endoscopic necrosectomy; $\mathrm{CO}_{2}$, carbon dioxide; ERCP, endoscopic retrograde cholangiopancreatography; PD, pancreatic duct; WON, walled-off necrosis.

*Two cases of mortality developed despite surgical intervention; ${ }^{\dagger}$ Others include air embolism, cutaneous fistula. 
18 patients, and gastric outlet obstruction in two patients. With respect to the DEN technique, transgastric (53 cases), transduodenal (five cases), or both (two cases) routes were used, and the multiple gateway technique was performed in 11 patients $(18.3 \%)$. Carbon dioxide $\left(\mathrm{CO}_{2}\right)$ gas was used instead of room air for insufflation in 54 patients (90\%). A median of 4 necrosectomy sessions (IQR, 2 to 6 sessions) was carried out and the median procedural time of each session was estimated to be 48 minutes (IQR, 37.25 to 64.23 minutes). Endoscopic retrograde cholangiopancreatography was performed in 27 patients and was followed by PD stenting in 17 patients (28.3\%). WON infection was identified in 44 patients (73.3\%) (Table 2). In terms of short-term outcomes, clinical success was achieved in 51 patients (85\%) and DEN complications developed in 21 patients (35\%). Among them, major complications such as bleeding requiring intervention $(n=9)$, perforation requiring surgery $(n=1)$, and air embolism $(n=1)$ developed in 10 patients $(16.7 \%)$. The WON recurrence was developed in four patients within 6 months after discharge, that might be attributed to unsuccessful DENs. Additionally, six patients underwent surgery and three patients succumbed to pancreatitis-related mortality (Fig. 1). Altogether, there were 16 patients $(26.7 \%)$ who displayed DEN failure or major complications associated with DEN. Multivariate analysis demonstrated that the only significant risk factor for those patients was an identified bacterial/fungal infection of the WON (odds ratio, 19.28; 95\% confidence interval, 1.50 to 261.7) (Table 3). Among 44 patients with positive microbial cultures, Candida infection was found in six patients and bacterial infection was noted in 41 patients. The bacterial infections included carbapenem-resistant Acinetobacter baumannii that was identified in five patients, carbapenemresistant Klebsiella pneumoniae in one patient, extendedspectrum beta-lactamase-producing Klebsiella pneumonia in one patient, methicillin-resistant Staphylococcus aureus in 11 patients, and vancomycin-resistant enterococci in two patients; infection with multidrug-resistant bacteria was found in 17 (28.3\%) of 60 patients (Table 4). Furthermore, at the end of the DEN procedure, complete clearance of necrotic tissue in the WON cavity under visualization of DEN was achieved in eight patients (13.3\%) (Fig. 2).

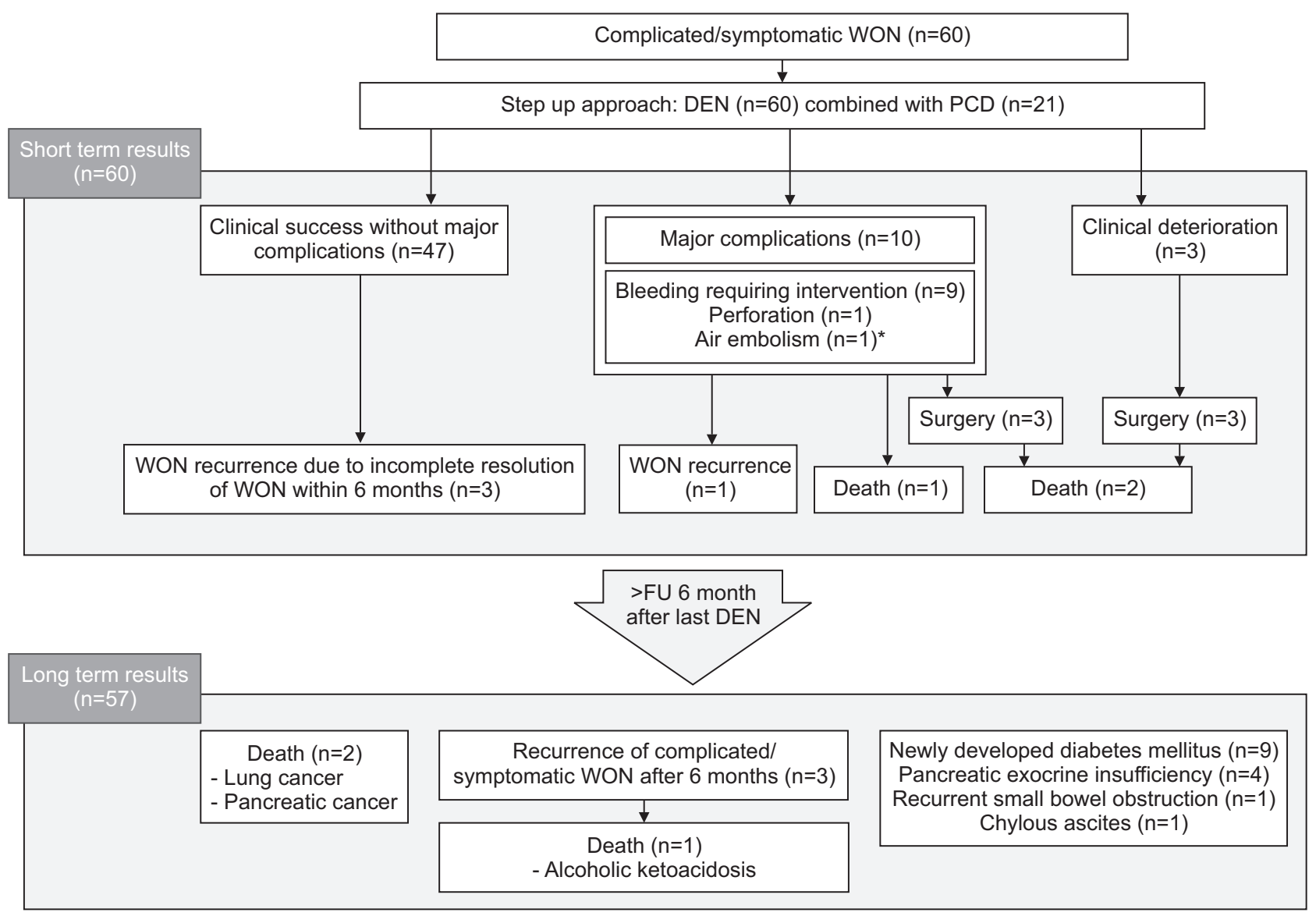

Fig. 1. Flowchart showing the treatment courses of the study patients.

WON, walled-off necrosis; DEN, direct endoscopic necrosectomy; PCD, percutaneous catheter drainage; FU, follow-up. *Air embolism and bleeding occurred simultaneously in one patient. 
Table 3. Risk Factors Associated with the Clinical Outcomes of Direct Endoscopic Necrosectomy

\begin{tabular}{|c|c|c|c|c|c|}
\hline Risk factor & $\begin{array}{l}\text { DEN failure* or with major } \\
\text { complications }^{+}(n=16)\end{array}$ & Successful DEN (n=44) & $p$-value ${ }^{\ddagger}$ & OR $(95 \% \mathrm{Cl})$ & $p$-value \\
\hline Age, mean $\pm S D$, yr & $43.9 \pm 14.2$ & $49.8 \pm 14.6$ & 0.016 & $0.99(0.94-1.04)$ & 0.679 \\
\hline Sex (male/female) & $14 / 2$ & $32 / 12$ & 0.314 & & \\
\hline $\mathrm{BMI}$, mean $\pm S D, \mathrm{~kg} / \mathrm{m}^{2}$ & $24.8 \pm 2.8$ & $24.2 \pm 4.0$ & 0.564 & & \\
\hline Identified WON infection (yes/no) & $15 / 1$ & 29/15 & 0.046 & $19.28(1.50-261.70)$ & 0.023 \\
\hline CRRT (yes/no) & $10 / 6$ & $4 / 40$ & 0.017 & $2.00(0.33-12.0)$ & 0.449 \\
\hline Additional PCD (yes/no) & 9/7 & $12 / 32$ & 0.064 & $3.30(0.71-15.34)$ & 0.128 \\
\hline $\begin{array}{l}\text { Initial drainage method } \\
\text { (plastic/metal stent) }\end{array}$ & $3 / 13$ & $16 / 28$ & 0.228 & & \\
\hline Gateway (single/multiple) & $12 / 4$ & $37 / 7$ & 0.462 & & \\
\hline Transpapillary PD stent (yes/no) & $2 / 14$ & $15 / 29$ & 0.120 & $0.22(0.35-1.35)$ & 0.218 \\
\hline $\begin{array}{l}\text { Pelvic extension of necrotic cavity } \\
\text { (yes/no) }\end{array}$ & $7 / 9$ & $8 / 36$ & 0.088 & $3.17(0.51-19.74)$ & 0.315 \\
\hline No. of cavity (multiple/single) & $11 / 5$ & $21 / 23$ & 0.242 & & \\
\hline $\begin{array}{l}\text { Largest diameter of necrotic cavity } \\
\qquad(\geq 15 \mathrm{~cm} /<15 \mathrm{~cm})\end{array}$ & $5 / 11$ & $27 / 27$ & 0.764 & & \\
\hline
\end{tabular}

DEN, direct endoscopic necrosectomy; OR, odds ratio; Cl, confidence interval; BMI, body mass index; WON, walled-off necrosis; CRRT, continuous renal replacement therapy; PCD, percutaneous catheter drainage; PD, pancreatic duct.

*Rescue surgery or death during index admission, readmission within 6 months; ${ }^{\dagger}$ Massive bleeding or perforation requiring radiological/surgical intervention or air embolism; ${ }^{\ddagger}$ Univariate analysis; ${ }^{\S}$ Multivariate analysis.

Table 4. Type and Frequency of Grown Organisms in Positive Aspirate/ Tissue Cultures

\begin{tabular}{lc}
\hline \multicolumn{1}{c}{ Variable } & Isolates, No. (\%) \\
\hline Total patients (monomicrobial/polymicrobial infection) & $44(35 / 9)$ \\
Total microorganisms & 64 \\
Gram negative & \\
Klebsiella pneumoniae & $6(9.4)$ \\
Pseudomonas aeruginosa & $2(3.1)$ \\
Enterobacter aerogenes & $5(7.8)$ \\
Escherichia coli & $1(1.6)$ \\
Acinetobacter baumannii & $9(14.1)$ \\
Citrobacter species & $2(3.1)$ \\
Aeromonas hydrophilia & $1(1.6)$ \\
Stenotrophomonas maltophilia & $6(9.4)$ \\
Morganella morganii & $1(1.6)$ \\
Gram positive & \\
Enterococci species & $7(10.9)$ \\
Staphylococcus aureus & $15(23.4)$ \\
Coagulase-negative Staphylococci & $2(3.1)$ \\
Streptococcus species & $1(1.6)$ \\
Fungus & \\
Candida albicans & $5(7.8)$ \\
Candida glabrata & $1(1.6)$ \\
Multidrug-resistant bacteria & 20 \\
ESBL-producing Klebsiella pneumoniae & $1(5.0)$ \\
Carbapenem-resistant Klebsiella pneumoniae & $1(5.0)$ \\
Carbapenem-resistant Acinetobacter baumannii & $5(7.8)$ \\
Methicillin-resistant Staphylococcus aureus & $11(55.0)$ \\
Vancomycin-resistant enterococci & $2(10.0)$ \\
\hline
\end{tabular}

ESBL, extended spectrum-beta lactamase.

\section{Long-term follow-up after DEN}

Complete follow-up data were obtained for 57 patients, after excluding patients that displayed early mortality in the study group. The median follow-up period was 27 months (IQR, 7.8 to 48.4 months). The recurrence of symptomatic/ complicated WON was observed in three patients (5.3\%) after 6 months of last session of DEN, and the median time to recurrence after completion of DEN was 193 days, 375 days and 433 days (Fig. 1). The cause of recurrence was alcohol in one and unknown in two patients. Long-term complications occurred in 14 patients (24.6\%) and included four cases of pancreatic exocrine insufficiency, nine cases of newly developed diabetes mellitus, one case of recurrent small bowel obstruction, and one case of chylous ascites (Table 5). Moreover, when the long-term outcomes and recurrences were compared according to the degree of DEN, there is no difference of those between visual estimation of complete and incomplete clearance of necrotic tissue in WON cavity at end session of DEN $(\mathrm{p}<0.99)$ (Table 6). In addition, three patients died due to causes unrelated to the DEN or pancreatitis: one succumbed to lung cancer after 63 months, one to alcoholic ketoacidosis after 26 months, and another patient died due to pancreatic cancer after 16 months.

\section{DISCUSSION}

With the evolution of endotherapy in recent years, endoscopic step-up approaches have gained popularity for the treatment of complicated or symptomatic WON. Various methods have been suggested, including step-up approaches in which DPPS or metal stents (e.g., tubular SEMS or lumen-apposing metallic stent) are placed to 

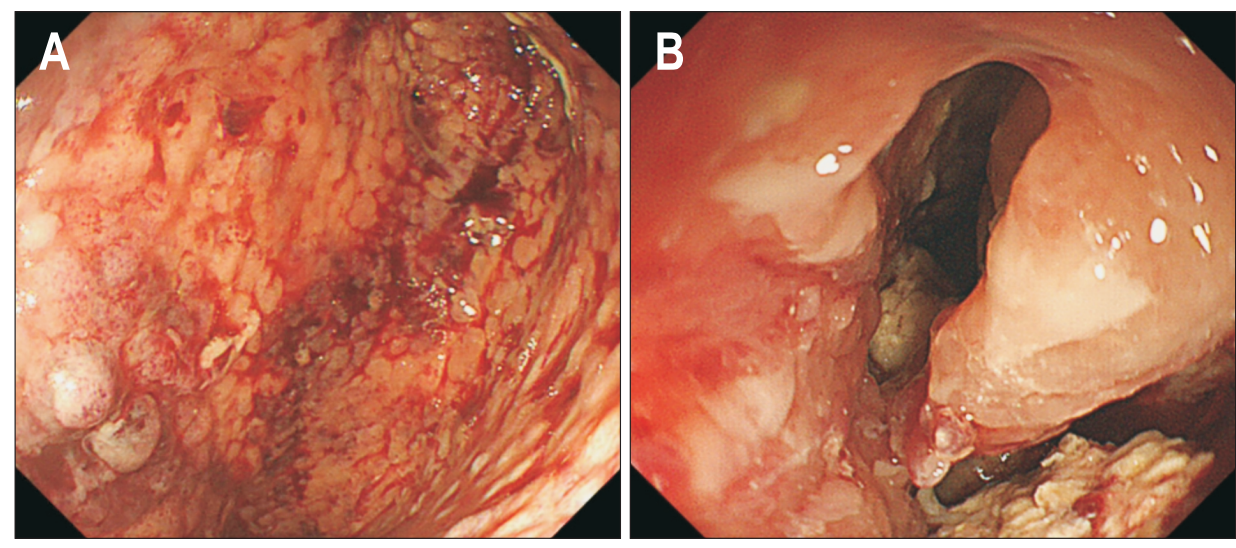

Fig. 2. Intracavitary image of a walledoff necrosis with (A) complete or (B) incomplete removal of necrotic debris in the last session of direct endoscopic necrosectomy.

Table 5. Long-term Follow-up of 57 Patients after Direct Endoscopic Necrosectomy

\begin{tabular}{lc}
\hline \multicolumn{1}{c}{ Outcome } & \multicolumn{1}{c}{ Value } \\
\hline $\begin{array}{ll}\text { FU duration after completion of endoscopic necro- } \\
\text { sectomy, median (IQR), mo }\end{array}$ & 27 (7.8-48.4) \\
Recurrence of complicated/symptomatic WON, No. (\%) & $3(5.3)$ \\
Recurrence time after completion of endoscopic & $378(193-433)$ \\
necrosectomy, median (range), day & \\
Long-term complication, No. (\%) & $14(24.6)$ \\
Pancreatic exocrine insufficiency & $4(7.0)$ \\
Newly developed DM & $9(15.8)$ \\
Others* & $2(3.5)$ \\
Death, No. $(\%)^{+}$ & $3(5.3)$ \\
\hline
\end{tabular}

FU, follow-up; IQR interquartile range; WON, walled-off necrosis; DM, diabetes mellitus.

*Others include chylous ascites and recurrent small bowel obstruction; ${ }^{\dagger}$ Alcoholic ketoacidosis, pancreatic cancer, and lung cancer.

drain the fluids and then if necessary, the remaining necrosis is removed by DEN. In general, the indications for intervention in the WON are as follows: (1) clinical suspicion or identified infection of the WON with clinical deterioration; (2) ongoing organ failure in the several weeks following onset of acute pancreatitis; (3) symptomatic sterile WON including intractable pain, persistent discomfort, and ongoing gastrointestinal and biliary obstruction; or (4) disconnected PD syndrome..$^{18}$ In this study, the major indication resulting in DEN is suspected infection of the WON in 40 patients (66.7\%). However, 44 patients (73\%) who underwent DEN had bacterial or fungal infection. In the 15 patients who reported intractable pain and the one patient with gastric outlet obstruction, infections were also identified. Additionally, of the 40 patients with suspected infections, pathogens were not confirmed in 12 patients. This is believed to result from preprocedural antibiotic use prior to the endoscopic treatment (Table 2). Currently, much debate surrounds the routine use of antibiotics in severe necrotizing pancreatitis, however antibiotic escalation could be beneficial for those requiring DEN because our study indicated that identified infection was the only significant risk factor associated with DEN failure and major complications. Furthermore, considering the high incidence of multidrug-resistant infection, appropriate antimicrobial therapy should be chosen in accordance with results from sensitive pathogen identifying tests. ${ }^{19} \mathrm{We}$ expect that this approach will result in a better DEN outcome.

Furthermore, we investigated whether long-term recurrence or complications may occur following DEN. Although long-term follow-up results were inadequate in previous studies, the 6-month follow-up data from the MISER trial ${ }^{15}$ suggested that new onset diabetes developed in six patients $(27.3 \%)$ and pancreatic insufficiency was diagnosed in 29 patients (85.3\%) following the endoscopic step-up approach for necrotizing pancreatitis. In the longterm follow-up data from the PANTER trial, ${ }^{10}$ which compared minimal versus open surgical necrosectomy, the step-up group had a lower proportion of incisional hernias ( $23 \%$ vs $53 \%$ ), pancreatic exocrine insufficiency ( $29 \%$ vs $56 \%$ ), and endocrine insufficiency ( $40 \%$ vs $64 \%$ ) after a mean follow-up of 86 months following discharge after the index admission. Additionally, the GEPARD study ${ }^{20} \mathrm{ob}-$ served that the DEN group had overall long-term clinical success in $84 \%$ of the patients. WON recurrence developed in $11 \%$ of the patients and new onset diabetes was diagnosed in $20 \%$ of the patients after a mean follow-up period of 43 months. In the present study, symptomatic/complicated WON recurrence was confirmed in $5.3 \%$ of the patients and long-term complications developed in $22.8 \%$ of the patients after a median follow-up period of 27 months. Specifically, new onset diabetes and exocrine insufficiency developed in $15.8 \%$ and $7.0 \%$ of the patients, respectively. This was comparable to the results of GEPARD study. Interestingly, we found DEN-related typical long-term complications of chylous ascites and intestinal obstruction that may have occurred during pancreatic surgery. Thus, it is necessary to take measures to minimize damage of viable 
Table 6. The Relationship between WON Recurrence (<6 Months vs $\geq 6$ Months) or Long-term Complications and the Degree of Clearance of Intracavitary Necrotic Tissue at the Last Session of DEN

\begin{tabular}{|c|c|c|c|}
\hline & \multicolumn{2}{|c|}{ Degree of clearance of intracavitary necrotic tissue at the end session of DEN } & \multirow[b]{2}{*}{ p-value } \\
\hline & $\begin{array}{c}\text { Complete clearance of intracavitary } \\
\text { necrotic tissue }(n=8)\end{array}$ & $\begin{array}{l}\text { Incomplete clearance of intracavitary } \\
\text { necrotic tissue }(n=49)\end{array}$ & \\
\hline $\begin{array}{l}\text { DEN failure of complicated/symptomatic } \\
\text { WON recurrence within } 6 \text { months after } \\
\text { discharge (yes/no) }\end{array}$ & $0 / 8$ & $4 / 45$ & $<0.99$ \\
\hline $\begin{array}{l}\text { Long-term WON recurrence more than } 6 \\
\text { months after discharge (yes } / \text { no) }\end{array}$ & $1 / 7$ & $2 / 47$ & 0.37 \\
\hline $\begin{array}{l}\text { Long-term complications including newly } \\
\text { developed DM, pancreatic exocrine } \\
\text { insufficiency, others (yes/no)* }\end{array}$ & $2 / 6$ & $12 / 37$ & $<0.99$ \\
\hline
\end{tabular}

WON, walled-off necrosis; DEN, direct endoscopic necrosectomy; DM, diabetes mellitus.

*Others include chylous ascites and recurrent small bowel obstruction.

pancreatic tissue as much as possible during DEN.

Multiple methods have been used to increase the effectiveness of necrosectomy, but many questions remain surrounding the uncertainty of optimal timing, number of sessions, interval between sessions, and the degree of completeness of DEN. ${ }^{1,6,21,22}$ To date, the endpoint of DEN has not been definitively established. These decisions should be based on patient's clinical course, the indication for intervention and endoscopist expertise and preference. Asian consensus statement suggested that the clearance of necrotic debris and pink granulation tissue lining the wall in the WON cavity were important parameters of successful DEN. ${ }^{23}$ However, it is also unclear whether necrosectomy should be carried out for complete debridement of necrotic tissue within the WON in every case because complete debridement may itself result in extensive injury of viable pancreatic parenchyma and possible damage to surrounding hidden blood vessels. If the patient's symptoms improve, it may be helpful to treat the underlying infection while maintaining transmural DPPS drainage without completely elimination of the necrotic tissues. In this study, complete clearance of necrotic debris in the WON cavity at the last session of DEN was only achieved in eight patients (13.3\%) under direct visualization. If the clinical resolution was achieved by a combination of appropriate antibiotic escalation with repeated DENs, the degree of necrotic tissue clearance in the WON at the last DEN session had no significant effects on symptomatic/complicated WON recurrence and long-term complications. In order to obtain better clinical outcomes, it is therefore recommended not only to perform microbial culture of both aspirated pus and necrotic tissue, but also to minimize unnecessary pancreatic injury during DEN.

There are several limitations to this study. First, the retrospective design and limited sample size have inherent limitations. This study was designed as a multicenter study to overcome size limitations, but each institution lacked consensus for the indication, timing, and DEN methods used. Furthermore, it would be ideal to have specific techniques and procedural elements for a successful DEN, but since various methods are used and inconsistent in almost all procedural details, a unified procedural technique cannot be presented. For instance, consensus has not been reached regarding the effectiveness of hydroperoxide or antibiotics lavage, access technique, amount of debridement, benefit of saline irrigation via nasobiliary tube, and use of preprocedural antibiotics or proton pump inhibitor during DEN. Secondly, for the evaluation of longterm complications, we defined exocrine and endocrine insufficiency based on the need for pancreatic enzyme replacement therapy and antidiabetics/insulin. This entails a risk of underestimating the number of patients with these insufficiencies, whereas direct pancreatic function testing or measurement of glycated hemoglobin or Cpeptide might have resulted in a more accurate evaluation. Thirdly, although various types of plastic stents and SEMS were used in this study, we could not identify the benefits of each stent type due to small sample size. Moreover, in Korea, of the currently available lumen-apposing metallic stents, only SPAXUS stent (Taewoong Medical) can be used, so the effectiveness of the $16 \mathrm{~mm}$ diameter Hot Axios (Boston Scientific) that has been used worldwide ${ }^{17}$ could not been verified. However, the results are considered to be meaningful, because incidental SEMS migrations is not uncommon during repeated endoscopic retrievals of necrotic tissue through the lumen of the SEMS. Finally, no standardized follow-up protocol was used for data collection and there were no objective criteria for visual assessing degree of necrotic debridement in the WON cavity. CT scans are an effective method for determining the size reduction of WON, so when determining the endpoint of DEN, it should be compared with the visual assessment of 
necrotic debris in the WON cavity. Unfortunately, no data has been collected in this study.

In conclusion, as a targeted and minimally invasive approach, DEN is regarded as an efficacious and reproducible technique for the treatment of complicated or symptomatic WON with an acceptable safety profile. Considering that long-term complications are similar to those observed after pancreatectomy, DEN should be performed meticulously so as to minimize damage to the pancreatic parenchyma and should be combined with antibiotics possessing adequate coverage against identified pathogens. Additional multicenter prospective studies are needed to validate the conclusions of our study.

\section{CONFLICTS OF INTEREST}

No potential conflict of interest relevant to this article was reported.

\section{AUTHOR CONTRIBUTIONS}

Conception and design of the study: J.H.C., S.S.L. Generation, collection, assembly, analysis and/or interpretation of data: Y.S.K., J.H.C., D.H.C., S.W.P., S.H.M., J.S.P., Y.N.L. Drafting or revision of the manuscript: Y.S.K., J.H.C. Approval of the final version of the manuscript: J.H.C., S.S.L.

\section{ORCID}

Yeon Suk Kim https://orcid.org/0000-0002-9060-5251 Jae Hee Cho https://orcid.org/0000-0003-4174-0091 Dong Hui Cho https://orcid.org/0000-0001-9003-9184 Se Woo Park https://orcid.org/0000-0003-1603-7468 Sung-Hoon Moon https://orcid.org/0000-0002-7879-3114 Jin-Seok Park https://orcid.org/0000-0001-9911-8823 Yun Nah Lee https://orcid.org/0000-0001-5588-784X Sang Soo Lee https://orcid.org/0000-0002-3785-2341

\section{REFERENCES}

1. Baron TH, DiMaio CJ, Wang AY, Morgan KA. American Gastroenterological Association clinical practice update: management of pancreatic necrosis. Gastroenterology 2020;158:67-75.

2. Banks PA, Freeman ML; Practice Parameters Committee of the American College of Gastroenterology. Practice guidelines in acute pancreatitis. Am J Gastroenterol
2006;101:2379-2400.

3. van Santvoort HC, Bakker OJ, Bollen TL, et al. A conservative and minimally invasive approach to necrotizing pancreatitis improves outcome. Gastroenterology 2011;141:12541263.

4. Jeon TJ, Lee KJ, Woo HS, et al. Refeeding syndrome as a possible cause of very early mortality in acute pancreatitis. Gut Liver 2019;13:576-581.

5. Lee KJ, Kim HM, Choi JS, Kim YJ, Kim YS, Cho JH. Comparison of predictive systems in severe acute pancreatitis according to the revised Atlanta classification. Pancreas 2016;45:46-50.

6. Arvanitakis M, Dumonceau JM, Albert J, et al. Endoscopic management of acute necrotizing pancreatitis: European Society of Gastrointestinal Endoscopy (ESGE) evidence-based multidisciplinary guidelines. Endoscopy 2018;50:524-546.

7. Yasuda I, Takahashi K. Endoscopic management of walledoff pancreatic necrosis. Dig Endosc 2021;33:335-341.

8. van Santvoort HC, Besselink MG, Bakker OJ, et al. A step-up approach or open necrosectomy for necrotizing pancreatitis. N Engl J Med 2010;362:1491-1502.

9. Cho JH, Kim YJ, Kim YS. Paradigm shift away from open surgical necrosectomy toward endoscopic interventions for necrotizing pancreatitis. Gastrointest Interv 2014;3:84-88.

10. Hollemans RA, Bakker OJ, Boermeester MA, et al. Superiority of step-up approach vs open necrosectomy in long-term follow-up of patients with necrotizing pancreatitis. Gastroenterology 2019;156:1016-1026.

11. Ross AS, Irani S, Gan SI, et al. Dual-modality drainage of infected and symptomatic walled-off pancreatic necrosis: longterm clinical outcomes. Gastrointest Endosc 2014;79:929935.

12. Yasuda I, Nakashima M, Iwai T, et al. Japanese multicenter experience of endoscopic necrosectomy for infected walledoff pancreatic necrosis: the JENIPaN study. Endoscopy 2013;45:627-634.

13. Gardner TB, Coelho-Prabhu N, Gordon SR, et al. Direct endoscopic necrosectomy for the treatment of walled-off pancreatic necrosis: results from a multicenter U.S. series. Gastrointest Endosc 2011;73:718-726.

14. van Brunschot S, van Grinsven J, van Santvoort HC, et al. Endoscopic or surgical step-up approach for infected necrotising pancreatitis: a multicentre randomised trial. Lancet 2018;391:51-58.

15. Bang JY, Arnoletti JP, Holt BA, et al. An endoscopic transluminal approach, compared with minimally invasive surgery, reduces complications and costs for patients with necrotizing pancreatitis. Gastroenterology 2019;156:1027-1040.

16. Bang JY, Wilcox CM, Arnoletti JP, Varadarajulu S. Superiority of endoscopic interventions over minimally invasive surgery for infected necrotizing pancreatitis: meta-analysis 
of randomized trials. Dig Endosc 2020;32:298-308.

17. Bang JY, Navaneethan U, Hasan MK, Sutton B, Hawes R, Varadarajulu S. Non-superiority of lumen-apposing metal stents over plastic stents for drainage of walled-off necrosis in a randomised trial. Gut 2019;68:1200-1209.

18. Trikudanathan G, Attam R, Arain MA, Mallery S, Freeman ML. Endoscopic interventions for necrotizing pancreatitis. Am J Gastroenterol 2014;109:969-981.

19. Lee HS, Lee SK, Park DH, et al. Emergence of multidrug resistant infection in patients with severe acute pancreatitis. Pancreatology 2014;14:450-453.

20. Seifert H, Biermer M, Schmitt W, et al. Transluminal endoscopic necrosectomy after acute pancreatitis: a multicentre study with long-term follow-up (the GEPARD Study). Gut 2009;58:1260-1266.

21. Trikudanathan G, Tawfik P, Amateau SK, et al. Early $(<4$ weeks) versus standard ( $\geq 4$ weeks) endoscopically centered step-up interventions for necrotizing pancreatitis. Am J Gastroenterol 2018;113:1550-1558.

22. Rerknimitr R. Endoscopic transmural necrosectomy: timing, indications, and methods. Clin Endosc 2020;53:49-53.

23. Isayama $H$, Nakai $Y$, Rerknimitr R, et al. Asian consensus statements on endoscopic management of walled-off necrosis. Part 2: endoscopic management. J Gastroenterol Hepatol 2016;31:1555-1565. 